\title{
DISPERSION MODEL INPUT PARAMETERS FROM NUMERICAL WEATHER PREDICTION OR SYNOPTIC OBSERVATIONS
}

\author{
Douglas R. Middleton \\ Met Office FitzRoy Road Exeter Devon EX1 3PB U.K.
}

\begin{abstract}
In the UK, local and regulatory dispersion modelling has usually been based upon input of hourly synoptic observations (OBS) from a not too distant station. Key variables arc wind speed and direction, cloud amount, and precipitation. Developments in weather forecasting mean that copious amounts of output data from numerical weather prediction (NWP) are produced at intervals, e.g. hourly or 3- hourly. These NWP data are available for use in air quality management. They can include friction velocity, heat flux and boundary layer depth, which arc not routinely measured. Operational air quality forecasts by the NAME III model rely on full 3-D NWP fields of data. Regulatory models need such data reduced to suit model input formats. Initial comparisons of NWP (at two grid scales, $\sim 60 \mathrm{~km}$ and $\sim 12 \mathrm{~km}$ ) and OBS data as used for dispersion models have been presented at the Harmo 11 Conference (Middleton, 2007). Weibull distributions of wind speed were fitted and the parameters compared. The present study describes the results from using parallel NWP and OBS data as input data to the meteorological pre-processor of the Met Office NAME III dispersion model. This processor is very similar in form and function to that of the ADMS model, in having similar boundary layer algorithms. Pre-processor results for dispersion parameters have been compared, namely friction velocity, heat flux, Monin Obukhov length and boundary layer depth. These parameters play a major role (along with source emissions and other factors) in influencing the dispersion calculations. The study compares values from NWP with pre-processor results using OBS data. It discusses potential advantages and disadvantages of NWP and OBS data in the pre-processors of regulatory dispersion modelling. The talk ends
\end{abstract} with a discussion of which NWP output variables might be best suited to managing air quality.

Key words: numerical weather prediction data, dispersion model, pre-processor, friction velocity, heat flux, stability.

\section{INTRODUCTION}

In the UK, dispersion modelling has usually been based upon input of hourly synoptic observations (OBS) from a not too distant station. Key variables are wind speed and direction, cloud amount, and precipitation. Developments in weather forecasting mean that copious amounts of output data from numerical weather prediction (NWP) are produced at intervals, e.g. hourly or 3-hourly. These NWP data are available for use in air quality management. They can include heat flux and boundary layer depth, which arc not routinely measured. Operational air quality forecasts by the NAME III model rely on full 3-D NWP fields of data. Regulatory models need such data reduced to suit model input formats. Initial comparisons of NWP (at two grid scales, $\sim 60 \mathrm{~km}$ and $\sim 12 \mathrm{~km}$ ) and OBS data as used for dispersion models have been presented at the Harmo 11 Conference by Middleton, (2007) who discussed wind speed, wind direction, total cloud cover, and temperature. Weibull

distributions of wind speed were fitted and the parameters compared.

The present study describes the results from using parallel NWP and OBS data as input data to the meteorological preprocessor of the Met Office NAME III dispersion model, Jones et al. (2007). This processor is very similar in form and function to that of the ADMS model, Carruthers et al. (1994) in having similar boundary layer algorithms. Pre-processor results for dispersion parameters have been compared, namely friction velocity, heat flux, Monin Obukhov length and boundary layer depth. These parameters play a major role (along with source emissions and other factors) in influencing the dispersion calculations. The study compares values from NWP with pre-processor results using OBS data for use in regulatory dispersion modelling.

\section{BOUNDARY LAYER DEPTH}

Middleton (2007) plotted frequency distributions of boundary layer depth arising from the Met Office Unified Model (UM) as used in the NAME III model. These were for NWP data at $12 \mathrm{~km}$ and $40 \mathrm{~km}$ grid scales \{ibid, Fig. 7). We have now used the NAME III met pre-processor to calculate boundary layer depths according to the synoptic data for wind speed and cloud cover. The distribution (not shown) has a much higher frequency of boundary layer depths $<100 \mathrm{~m}$ being calculated from the OBS data than was seen in the earlier study for NWP data. Consequently there is a significant change to the distribution here - shallow mixing depths are much more prevalent when using synoptic OBS data (as has been common UK practise) with the NAME III or ADMS met pre-processors than if the NWP depths are used. This will alter both pollutant trapping and ground level exposure from elevated plumes vis a vis NWP depths.

\section{ATMOSPHERIC STABILITY}

Atmospheric stability depends on the relative importance of the convection (sensible heat flux) and the turbulent drag (friction velocity). The Monin Obukov Length $L \mathrm{~m}$ is a measure of their relative importance and is the stability parameter used in current dispersion models. In the ADMS and NAME III met pre-processors, the constants below give:

$$
\mathrm{L}=-\frac{\rho C_{p} T}{\kappa g} \frac{u_{*}^{3}}{H}=-91065.6 \frac{u_{*}^{3}}{H}
$$


Where density of the atmosphere $\rho=1.225 \mathrm{kgm}^{-3}$, specific heat of air at constant pressure $C_{p}=1012.0 \mathrm{JK}^{-1} \mathrm{~kg}^{-1}$, absolute temperature of the air $T=288.15$ Kelvin, von Karman's constant $\kappa=0.4$, acceleration due to gravity $g=9.80665 \mathrm{~ms}^{-2}$; the sensible heat flux is $H \mathrm{Wm}^{-2}$ and the friction velocity cubed is $u_{*}^{3} \mathrm{~m}^{3} \mathrm{~s}^{-3}$. The combined constants denoted konst equals $91065.6 \mathrm{kgm}^{-2}$. The ratio $u_{*}^{3} / H$ has units $\mathrm{m}^{3} \mathrm{~kg}^{-1}$. Here a new method to evaluate the frequency distribution of the stability parameter is presented and used to compare NWP and OBS derived data sets.

\section{Dependence of $L$ upon $H$ and $\boldsymbol{u}$ *}

When analysing a year of met data from different sources, observations (OBS) or numerical weather prediction (NWP), a simple method of plotting the values of $L$ is required. However stability $L$ depends upon two physical parameters, the sensible heat flux $H$ and the friction velocity $u *$ for momentum. These quantities can be directly measured using turbulence instrumentation (sonic anemometers and fast response thermometry), and are easily related mentally to the prevailing meteorological conditions, such as is it windy, is it sunny, is it a clear night. One of the long-standing advantages of the old Pasquill (1961) stability scheme has been its simplicity as a mental model of how such observable features might influence plume dispersion. The Monin Obukhov length is a derived quantity that users of dispersion models find less easy to have a simple mental model of how it relates to meteorological conditions. Golder (1972) provided a method for relating the two methods - their relationship varies with surface roughness. In the present paper we show a method to plot the $\sim 8760$ hourly values for a year of stability data, so that differences in the stabilities within data sets of $L$ can be quantified, and the relative effects of differences in sensible heat flux and/or friction velocity be explained. We consider how such differences, once identified, might be related back to the boundary layer physics of the NWP model, to site characteristics, or to instrument limitations in the OBS data. The aim of the new plots described here is to provide a physically interpretable picture or "stability map" of the stability distribution. We have found this method to be a useful tool to provide useful insights into the evaluation of NWP versus OBS data for regulatory dispersion modelling when analysing the stability regime.

\section{Data Processing}

In this study we have three sources of data:

- Hourly synoptic observations - retrieved from the archive every hour at each station and year of study (2004), and written into ADMS format.

- NWP mesoscale $(12 \mathrm{~km})$ data - Analysis and Forecast fields were interpolated every hour to each site latitude/longitude using interpolation subroutines within NAME III model, and written into ADMS format.

- NWP regional $(40 \mathrm{~km})$ data - Analysis and Forecast fields were interpolated every hour to hourly values at each site latitude/longitude using interpolation subroutines within the NAME III model, and written into ADMS format. Regional data are cut out from global NWP model output to conserve archive space.

Analysis fields may be every three or six hours; Forecast fields are used at intervening times. The effective roughness length used within the UM to create a representative drag during forecasting is not easily defined, and we have not investigated this aspect of using NWP data for dispersion modelling; further work is needed. The NAME III model met pre-processor was run on each ADMS format file, using $z_{0}=0.1 \mathrm{~m}$ at all the locations here, and the resulting NAME III output results plotted below. For the OBS data, the pre-processor calculated the heat flux, friction velocity and boundary layer depth in the usual manner using wind speed, cloud cover, and time of day. For the NWP data, heat flux and boundary layer depths were available from the UM, so were interpolated to the site position and used without further calculation, though sometimes constrained by limits within the pre-processor. NAME III can use (if present) or estimate (if absent) these quantities.

\section{STABILITY MAPS: PLOTTING HVERSUS $u$ *}

Figure 1 uses data from a well exposed inland station at Brize Norton, an airfield in the rolling Oxfordshire countryside to show scatter plots of heat flux $H$ versus friction velocity $u *$ as follows: Figure 1 (a), synoptic OBS; 1 (b) NWP-mesoscale; and 1 (c) NWP-regional data. We superimposed a series of isopleths for arbitrarily chosen (convenient) values of $L$ on the plot. The isopleths are curved because the equation for $L$ depends upon $u_{*}^{3}$.
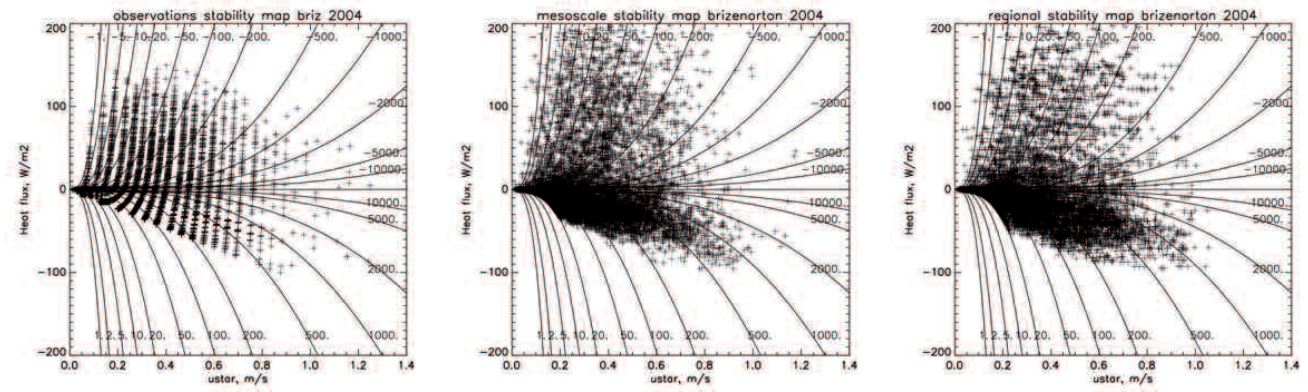

Figure 1. Stability map: Brize Norton, 2004, (a) OBS data (b) NWP mesoscale (12 km) data (c) NWP regional (40 km) data. 
Transformation of the horizontal axis to $u_{*}^{3}$ produces linear isopleths of constant $L$; see Figure 2, with the same data as in Figure 1 (Brize Norton; 2004), but now with axes of heat flux $H$ versus friction velocity cubed $u *{ }^{3}$. This simplifies the analysis that will be developed below based upon our geometrical construction of straight $L$-isopleths. However notice in Figure 2 that the non-linear horizontal axis has had the undesired effect of compressing many of the points nearer to the origin. Figures 1 and 2 make it possible to see the joint distribution of heat flux and friction velocity that together influence the stability and hence the rate of turbulent mixing in the lower atmosphere. However it is difficult from these scatter plots to get a feel for the frequencies with which particular values of $L$ occur. This is because with 8750 points (one year) many fall on top of each other. It is hard to see for example over what proportion of the time the stability was diagnosed as stable or as unstable. It is interesting to see the patterned effect in Figures 1(a) and 2(a) showing that the observations data are more discontinuous, reflecting the way in which cloud cover (oktas) and especially wind speed $\left(\mathrm{ms}^{-1}\right)$ are reported.
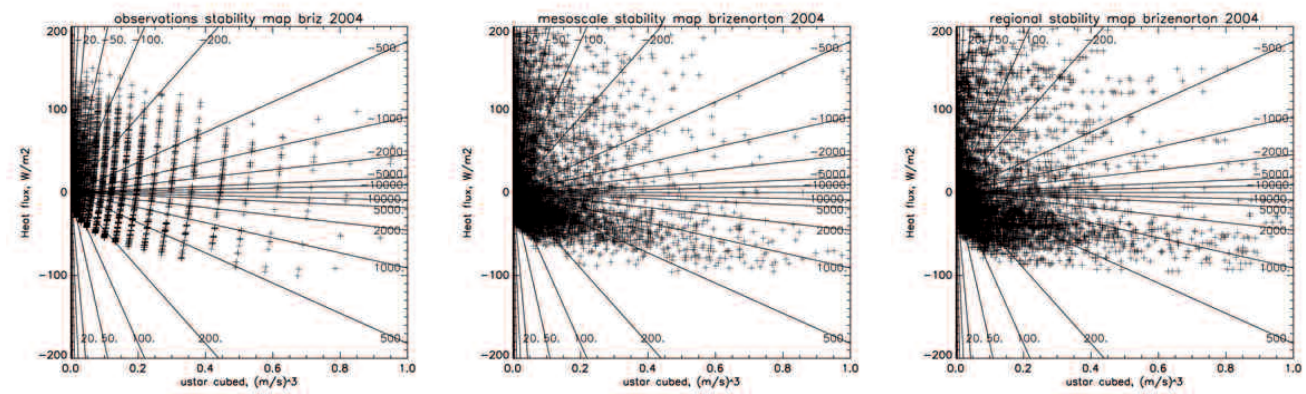

Figure 2. Stability map with $u *{ }^{3}$ axis: Brize Norton, 2004, (a) OBS data (b) NWP mesoscale (12 km) data (c) NWP regional (40 km) data.

\section{Calculation of Regular Isopleths for stability $L$}

In Figure 2, counting the number of points that lie between each isopleth for $L$ would give an indication of the frequency distribution of $L$. The isopleths in Figure 2 were drawn at arbitrary intervals for convenience. However for use as the bin boundaries in sorting the $L$ data into a histogram, we require the isopleths to be regularly spaced. It is convenient to do this by choosing the lines at equally spaced angles on $H$ versus $u_{*}{ }^{3}$. Angle $\alpha$ is defined starting at $-\pi / 2$ and stepping in equal angular increments $\Delta \alpha$ to $\pi / 2$. These lines are centred at the origin of the graph $H=0.0$ $u *{ }^{3}=0.0$. The values of $L$ on the edges of each sector are the bin limits for the histogram and are calculated as follows.

\section{Values for the Stability Subscript Index $a$}

To go from stable to unstable on the graph, choose $n$ angular steps, of width $\Delta \alpha=\pi / n$, from $-\pi / 2$ to $\pi / 2$. For index $a$, stepping from 0 to $n$, the corresponding angle $\alpha$ is

$$
\alpha=-\pi / 2+a \Delta \alpha=-\pi / 2+a \pi / n .
$$

From Equation (1), on a graph of $H$ versus $u_{*}^{3}$, the slope $m=\tan \alpha=Y / X$ (where the distances on the graph are $Y$ and $X$ ) is given by $H / f u_{*}{ }^{3}$. The factor $f$ is required in order to allow for the different limits on the two axes. In our case the factor was as follows:

$$
f=\frac{H_{2}-H_{1}}{\left(u_{*}^{3}\right)_{1}-\left(u_{*}^{3}\right)_{2}}
$$

with the scale limits being $H_{1}=-200.0$ to $H_{2}=+200.0 \mathrm{Wm}^{-2}$ in the vertical, and $\left(u_{*}^{3}\right)_{1}=0.0$ to $\left(u_{*}^{3}\right)_{2}=1.0 \mathrm{~m}^{3} \mathrm{~s}^{-3}$ in the horizontal, giving a value of the factor of $f=400.0 \mathrm{Wm}^{-5} \mathrm{~s}^{3}$. At each angle $\alpha$,

$$
L=-\frac{91065.6}{f \tan \alpha}
$$

Summarising, each integer value of the subscript $a$ is used to calculate the angle $\alpha$ and corresponding values for the bin intervals $L_{a-1}$ to $L_{a}$ on the Monin Obukhov stability length. The bin limits are used to sort the data, as below.

\section{Bin Limits on $\boldsymbol{L}$ for Sorting Stability Data}

As subscript $a$ is increased in linear steps, $L_{a}$ moves in the desired manner from strongly stable through neutral to strongly unstable, changing sign and traversing the discontinuity at neutral stability. The index or subscript $a$ is a convenient surrogate variable for sorting or plotting $L_{a}$. Whilst the angular increments of $\alpha$ are of equal width, and the sectors on the graph into which points are counted are equi-spaced, the arithmetic sequence of successive values for the bin limits on $L$ are somewhat unusual (not shown). We used the limits $L_{a}$ using $n=45$ ( $a=0$ to $a=45$ ) and $f=400.0$ in the present work to sort the year's hourly data and to plot a frequency distribution of $L$. These plots are a convenient tool for comparing the stabilities at different sites, and for comparing OBS data with NWP-mesoscale and with NWP-regional (global) data. Since the method was derived geometrically, it also means the distribution can easily be related to how the points fall on the scatter plot of $H$ versus $u_{*}^{3}$, where the isopleths are straight lines. 
In coding the method, missing data must be excluded, but in Equation 1 division by $H$ means that $L$ can take any arithmetic value. Typical codes like -999.9 to designate missing values of $L$ cannot be used. Therefore when sorting, we count valid data into bins only when both $H$ and $U_{*}$ have valid data. For $L_{a-1}<L_{a}$ the counter is increased if $L_{a-1} \leq L_{x}<L_{a}$. In the central sector which spans neutral stability, the bin limits change sign when $L_{a-1}>L_{a}$; this is a special case and the data are counted into the bin provided $L_{a-1} \leq L_{x} \leq+\infty$ or $-\infty \leq L_{x} \leq L_{a}$.
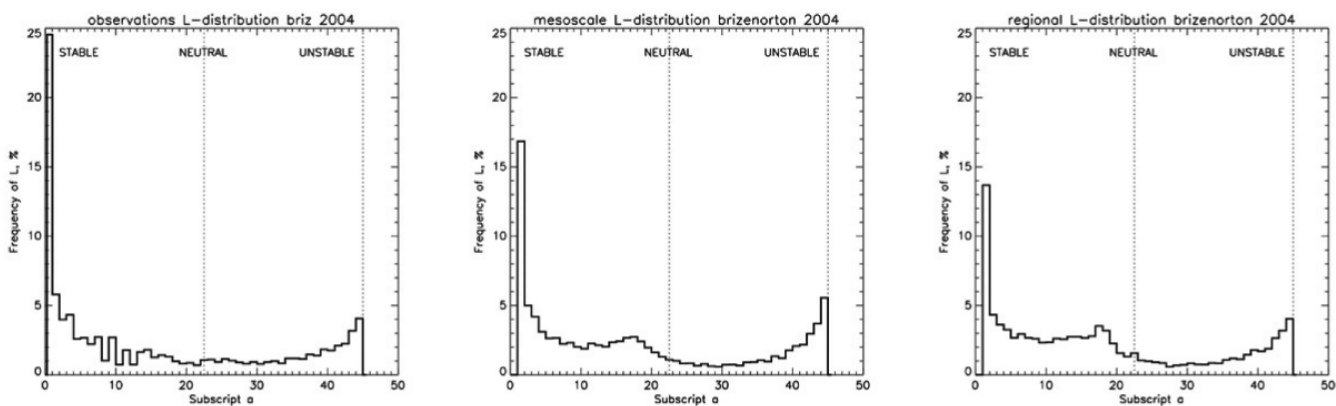

Figure 3. $\boldsymbol{L}$-Distribution: Brize Norton, 2004, (a) OBS data (b) NWP mesoscale (12 km) data (c) NWP regional (40 km) data.

\section{Pasquill Stability Categories}

Pasquill (1961) introduced a stability classification scheme, which sorted meteorological conditions into a small number of classes, useful because they relied on existing synoptic data for wind speed, insolation, cloud cover, and day or night. In the absence of any nearby weather fronts it could be used for a rapid assessment of the likely rate of spreading of a plume. In the Pasquill (1961) scheme, Classes A to CD were unstable, Class D was neutral stability and Classes E and F were stable. Later a more stable Class G was added. Smith (1979) revised these classes developing simple continuous numerical equivalents on a scale from 0 to 7 , where $P=0$ to 1 was A (unstable) $P=3.6$ was D (neutral) and $P=6$ to 7 was G (stable). Golder (1972) proposed relations between stability parameters, including Pasquill Classes, Monin Obukhov length, and Richardson number, with some dependence upon roughness length $z_{0}$ We used a regular geometrical sub-division of the $H$ versus $u *{ }^{3}$ domain, and whilst this covers all possible stabilities, it does not necessarily imply any regular steps in plume spreading. Further work is needed to see how plume spreading might increase as the angle $\alpha$ is varied according to Section 2.1. This could be calculated for given $z_{0}$ with the values of $L_{a}$ using Equations 2, 3 for $\sigma_{\phi,} \sigma_{\Theta}$ in Golder (1972) followed by Equations 1, 2 for $\sigma_{y} / x$ and $\sigma_{z} / x$ in Pasquill (1961). Figure 4 in Golder (1972) suggests that Pasquill Class D for $z_{0}=0.1$ encompasses values of $L$ from -26 to $+27 \mathrm{~m}$, or many of our stability bins, showing the sub-division is quite different. Whilst easy to visualise, our approach would not be expected to correspond directly in terms of values of $L$ to the old Pasquill and Smith schemes. Pasquill's concept was that the different Classes represented significant measured differences in the spreading of experimental plumes.

\section{RESULTS}

The main results were obtained by plotting the two key variables, namely sensible heat flux and the friction velocity, that are required to calculate the stability parameter Monin Obukhov length $L$. This scatter plot is based upon a simple physical understanding that to interpret differences in met data that are relevant to how they may affect dispersion modelling, it was necessary to have a visual feel for how the hourly data points fall. We have denoted this scatter plot a stability map and added to it the isopleths of constant $L$ in linear form as well as curved. A simple method to calculate incremental bin limits for $L$ was used for sorting hourly data into a frequency distribution - this is plotted here as a histogram of frequency of $L$ versus the subscript $a$ so that the plot reads naturally from most stable through neutral to most unstable. Comparison of stability maps and the $L$-distributions at a range of sites has shown that their NWP results can be classified into inland sites and coastal sites.

- At inland sites (e.g. Figures 1-3) both scale of NWP tested here, $12 \mathrm{~km}$ or $40 \mathrm{~km}$, provide an adequate representation of the stability regime.

- At coastal sites however (e.g. Figures $4-5)$ the coarser NWP regional (40 km) data were much less representative of the observations based stability regime.

- In complex terrain Aviemore is behaving largely as an inland site, but with lighter winds due to the drag.

- Estuary sites show a mixed stability behaviour, probably depending on where the site is located relative to local geography.

The coastal sites are probably best represented by NWP mesoscale $(12 \mathrm{~km})$ data, rather than the coarser data that seems at some sites (not shown here) to include too strong a sea based moderation of the heat flux, and thus too little dynamic range of stability, with a preponderance of neutral stabilities which is not seen in the pre-processed observations and NWP mesoscale $(12 \mathrm{~km})$ results. Extremes in air pollution thus may not be adequately represented 
by the coast if the regional $(40 \mathrm{~km})$ data are used. It appears much better at these stations based on the results here to elect to use NWP mesoscale data at the coast - at inland sites the choice seems much less critical.
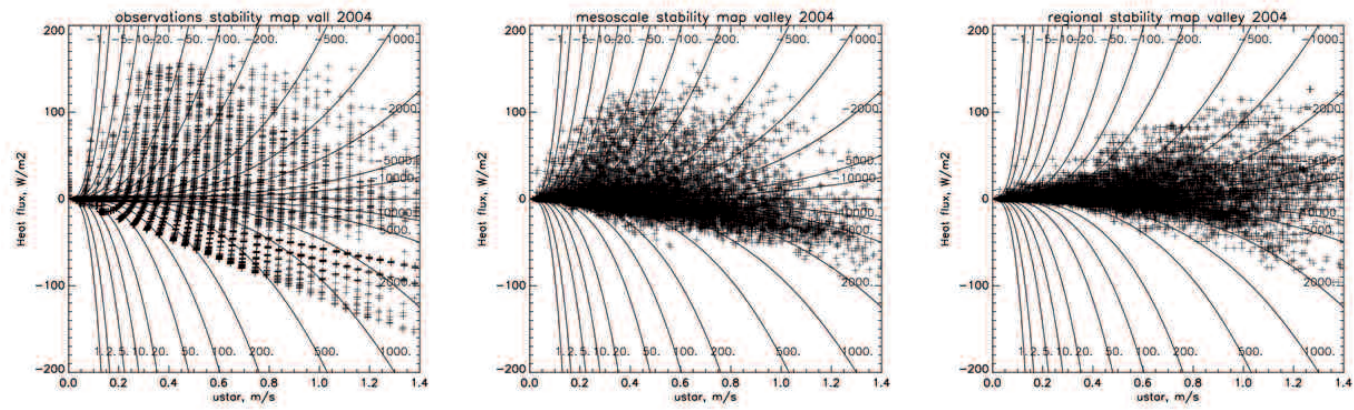

Figure 4. Stability map: Valley, 2004, (a) OBS data (b) NWP mesoscale (12 km) data (c) NWP regional (40 km) data.
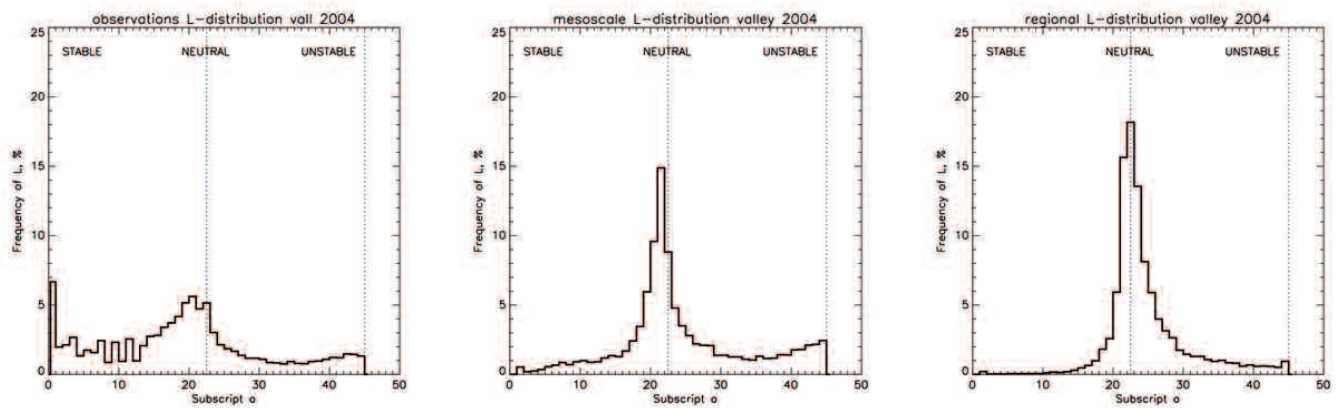

Figure 5. $\boldsymbol{L}$-Distribution: Valley, 2004, (a) OBS data (b) NWP mesoscale (12 km) data (c) NWP regional (40 km) data.

\section{CONCLUSIONS}

Boundary layer depths derived by the met pre-processor from OBS data have a high abundance of low values $<100$ $\mathrm{m}$, whereas those in NWP data from the UM have a much lower frequency of shallow depths.

Scatter plots of $H$ versus $u_{*}^{3}$ have linear isopleths of $L$ and have been used to derive bin limits for a regular set of bins to sort the data into a stability frequency distribution. Differences between OBS and NWP derived dispersion parameters were evident, but varied according to the type of location. A number of sites were studied (but not shown here).

As well as comparing OBS and NWP, the method used here has also been used to compare NWP model grid size effects, by comparing $12 \mathrm{~km}$ and $40 \mathrm{~km}$ data. With the coarser grid, coastal stations tended more often to be neutral than at inland sites.

\section{REFERENCES}

Clarke, R. H. et al., 1979: A model for short and medium range dispersion of radionuclides released to the atmosphere.

Report NRPB-R91, National Radiological Protection Board, Harwell, Didcot, Oxon OX11 0RQ, UK.

Golder, D., 1972: Relations among stability parameters in the surface layer. Boundary-Layer Meteorol., 3, 47-58.

Middleton, D. R., 2007: Meteorological data from numerical weather prediction or observations for dispersion modelling. Presented at 11th Int. Conf. on Harmonisation within Atmospheric Dispersion Modeling for Regulatory Purposes. 2-5 July 2007, Queen's College, Cambridge, U.K. Submitted to IJEP (under review)

Pasquill, F., 1961: The estimation of the dispersion of windborne material. The Meteorological Magazine, 90, 33-49.

Smith, F. B., 1979: Diffusion in the lower layers of the atmosphere. Appendix A, pages 60-65, published in report NRPB-R91 in Clarke et al. (1979) above.

Carruthers, D.J., Holroyd R.J., Hunt J.C.R., Weng W.S., Robins A.G., Apsley D.D., Thomson D.J. and Smith F.B., 1994: UK-ADMS - a new approach to modelling dispersion in the Earth's atmospheric boundary layer, Journal of Wind Engineering and Industrial Aerodynamics, 52, 139-153. See also www.cerc.co.uk.

Jones, A.R., Thomson, D.J., Hort, M. and Devenish, B, 2007: The U.K. Met Office's next-generation atmospheric dispersion model, NAME III, Air pollution modelling and its application XVII. Borrego and Norman (editors), Springer. 